\title{
How to achieve synergy between medical education and cognitive neuroscience? An exercise on prior knowledge in understanding
}

\author{
Dirk J. Ruiter • Marlieke T. R. van Kesteren • Guillen Fernandez
}

Received: 15 April 2010/Accepted: 16 August 2010/Published online: 31 August 2010

(C) The Author(s) 2010. This article is published with open access at Springerlink.com

\begin{abstract}
A major challenge in contemporary research is how to connect medical education and cognitive neuroscience and achieve synergy between these domains. Based on this starting point we discuss how this may result in a common language about learning, more educationally focused scientific inquiry, and multidisciplinary research projects. As the topic of prior knowledge in understanding plays a strategic role in both medical education and cognitive neuroscience it is used as a central element in our discussion. A critical condition for the acquisition of new knowledge is the existence of prior knowledge, which can be built in a mental model or schema. Formation of schemas is a central event in student-centered active learning, by which mental models are constructed and reconstructed. These theoretical considerations from cognitive psychology foster scientific discussions that may lead to salient issues and questions for research with cognitive neuroscience. Cognitive neuroscience attempts to understand how knowledge, insight and experience are established in the brain and to clarify their neural correlates. Recently, evidence has been obtained that new information processed by the hippocampus can be consolidated into a stable, neocortical network more rapidly if this new information fits readily into a schema. Opportunities for medical education and medical education research can be created in a fruitful dialogue within an educational multidisciplinary platform. In this synergetic setting many questions can be raised by educational scholars interested in evidence-based education that may be highly relevant for integrative research and the further development of medical education.
\end{abstract}

D. J. Ruiter $(\bowtie) \cdot$ M. T. R. van Kesteren

Department of Anatomy, Radboud University Nijmegen Medical Center, Route 109,

P.O. Box 9101, 6500 HB Nijmegen, The Netherlands

e-mail: d.ruiter@pathol.umcn.nl

D. J. Ruiter · M. T. R. van Kesteren - G. Fernandez

Donders Institute for Brain, Cognition, and Behavior, Donders Center for Cognitive Neuroimaging

Nijmegen, Radboud University Nijmegen Medical Center, Nijmegen, The Netherlands

G. Fernandez

Department of Neurology, Radboud University Nijmegen Medical Center, Nijmegen, The Netherlands 
Keywords Cognitive neuroscience $\cdot$ Evidence-based education - Medical education Prior knowledge $\cdot$ Reflections $\cdot$ Research agenda $\cdot$ Schema $\cdot$ Synergy

\section{Introduction}

Cognitive neuroscience attempts to understand how knowledge, insight and experience are processed in the brain and to clarify their neural correlates as demonstrated by various electrophysiological and imaging techniques (Goswami 2006). There are many reasons why cognitive neuroscience should convey important information to and interact with education (TLRP Commentary 2008). First of all, the brain is the principal organ involved in learning. Secondly, improving education, especially in children, is a priority in many countries. Thirdly, increased knowledge of brain function can inform and improve educational practice. Fourthly, neuroscience is rapidly progressing which is accompanied by a great momentum. Fifthly, and quite relevant, a scientifically based approach may prevent the introduction of questionable educational practices in the classroom (TLRP Commentary 2008). Sixtly, a multimedia revolution is going on that will enable further probing and promoting of a broad spectrum of human learning (Quellmalz and Pellegrino 2009). Seventhly, a new generation of learners is emerging that should survive the technological alterations of the modern mind (Small and Vorgan 2008). In light of this overwhelming rationale it is paradoxical that cognitive neuroscience so far has had only limited influence on education, let alone medical education. A plausible explanation for this phenomenon is the presence of gaps between cognitive neuroscience and education. A major challenge therefore is how to fill these gaps and achieve synergy between medical education and cognitive neuroscience.

During the past 30 years fruitful interaction between education and cognitive neuroscience has been hampered by the presence of gaps. Bruer (1997) only a decade ago even stated that this interaction was a bridge too far. From the relevant literature (Varma et al. 2008; Perkins 2009) two gaps emerge, i.e. one between (cognitive) neuroscience and education, and another between practice of education and educational research. The first gap mentioned is based on different levels of abstraction between (cognitive) neuroscience and education, and epistemical differences between explanation theories and action theories. The latter represents the contrast between basic science on the one hand and practicing science on the other hand (Perkins 2009). These involve scientific concerns, i.e. in-principle differences in methods, data, theory, and philosophy (Varma et al. 2008). Another set of concerns is pragmatic, i.e. consideration of costs, timing, locus of control, and likely payoffs (Varma et al. 2008). Furthermore, over-simplification of findings from neuroscience, over-interpretation their outcome and premature introduction into the classroom have given rise to so called "neuromyths" that subsequently yielded an adverse effect on the interaction between cognitive neuroscience and education (Goswami 2006; Della Salla 2009). Despite the presence of these obstacles several prominent researchers, both from cognitive neuroscience and education, currently believe that bridging the gaps is possible, leading to a fruitful mutual interaction, i.e. synergy (Goswami 2006; Varma et al. 2008; Perkins 2009; Della Salla 2009; Ansari and Coch 2006).

Recently a number of constructive approaches has been proposed in order to be able to bridge the gaps mentioned. As the gaps include scientific and pragmatic aspects, successful bridging involves a complex and multi-step approach. According to Ansari and Coch (2006) a concerted effort is needed to think about the goals and benefits of connecting 
education and cognitive neuroscience. This is currently undertaken in the new field of mind, brain, and education (MBE), that should provide realistic information about the potential outcomes of interactions between cognitive neuroscience and education (Coch and Ansari 2009). Coch and Ansari strongly propose to train teachers in neuroscience and to train cognitive neuroscientists in basis educational theory and methodology in order to reach a proper integrative approach (Coch and Ansari 2009). Mason advocates bridging, at least as the first step, by educational psychology (Mason 2009). Hereby a two-way path is foreseen in which educational psychologists are the integrative scholars as indicated by Coch and Ansari (Coch and Ansari 2009). In this two-way path the initiating role of educationalists should be strong, raising salient issues and questions for neuroscientific research (Christodoulou and Gaab 2009). It was even suggested by Greenwood (2009) and Meltzoff et al. (2009) that educators, together with those working in other disciplines, develop the science of teaching, or the new science of learning, respectively. The new science of learning arises from several disciplines, i.e. developmental psychology, machine learning, neuroscience and education and it has great promise for transforming educational practices (Meltzoff et al. 2009). This proposal is in line with the earlier mentioned new field of MBE and educational psychology, but it is more comprehensive and ambitious. In order to further elucidate the potential benefits of this integrative approach two considerations are important (De Jong et al. 2008). Firstly, it should be explored how educational principles, mechanisms, and theories could be extended or refined based on findings from cognitive neuroscience. Secondly, it should be explored which neuroscience principles, mechanisms, or theories may have implications for educational research and could lead to new interdisciplinary research ventures. A recent report on explorations in learning and the brain (De Jong et al. 2008) concludes that (a) the time has come to design new types of research that will provide us with adequately detailed and applicable guidelines for educational design based on neuroscientific data, and (b) a starting point is given for an agenda for educational science research that incorporates neuroscientific theories and techniques. Fruits of a constructive dialogue between cognitive neuroscience and education include a common language and understanding about learning, more educationally focused scientific inquiry, and multidisciplinary research projects (TLRP Commentary 2008).

Much of the foregoing considerations on education can be directly translated to medical education. Medical education beside it has its own dimensions such as particular educational characteristics, preclinical and clinical learning environments, a culture (attitude) of strong commitment, and organizational dynamics and constraints (Eva 2005; Michael 2006; Neville and Norman 2007; Schmidt and Rikers 2007; Schuwirth and Van der Vleuten 2006). In addition, since medical neuroscience (e.g. neuroanatomy, neurophysiology, clinical neurology, medical psychology) is an important part of the medical curriculum many opportunities for interactions with medical education are available, as noted earlier by other authors (Schmidt et al. 1990). Highly interesting research topics from a view point of medical education, that also could have relevance for implementation in educational practice, include the importance and mechanisms of prior knowledge for learning, the effects and mechanisms of multimodal learning, the effects and mechanisms of learning metacognitive and regulative skills, and the optimal development of expert know-how (De Jong et al. 2008). As the focus of this paper is on the question how to achieve synergy between medical education and cognitive neuroscience we would like to give an in-depth discussion on only a few of the previously mentioned research topics as an example and not present a more global discussion on several topics. For this purpose we have chosen the topic of the importance and mechanisms of prior knowledge for understanding, based on its great promises for medical education and medical education research 
(Michael 2006; Patel et al. 2008), and our own current research interest (Takashima et al. 2006, 2009; van Kesteren et al. 2010a). We first will discuss basic aspects of prior knowledge from cognitive psychology, subsequently make a translation to cognitive neuroscience, followed by a discussion on the opportunities for medical education and medical education research. Finally our conclusions and recommendations how to stimulate the synergy between medical education and cognitive neuroscience in a broader perspective are presented.

\section{Insights from cognitive psychology on prior knowledge in understanding}

A critical condition for the acquisition of new knowledge is the existence of previously acquired relevant knowledge, i.e. of a well developed mental model or schema (Bransford and Johnson 1972; Alba and Hasher 1983). The term schema is most often used to refer to the general knowledge a person possesses about a particular domain. Weaving the strands of information into a coherent schema will facilitate students' understandings of content (Bransford 1983). A schema allows for the encoding, storage, and retrieval of information related to that domain (Alba and Hasher 1983). Alba and Hasher propose a modal theory by which schema-driven encoding of complex information is characterized by four basic processes: selection and reconstruction, abstraction, interpretation, and integration (Alba and Hasher 1983). They consider reconstruction as the central retrieval process of schema theory, and reduction of the amount of information as an important element, but recognize that memory representation is far richer and detailed than schema theory would suggest. Based on recent social and cultural perspectives McVee et al. (2004) have revisited schema theory and as a result thereof consider schemas as transactional and embodied constructs. They view schema and other cognitive processes or structures as embodied, i.e. who we are as biological beings determines our sensorial interactions with the world and thus the nature of the representations we construct. Further, they consider knowledge to be situated in the transaction between world and individual. Finally, according to these authors these transactions are mediated by culturally and socially enacted practices carried out through material and ideal artifacts. Following their interpretation, a schema therefore has an embodied, transactional and cultural nature. This interpretation has implications for the definition, the formation, the processing, and transformation of schemas (McVee et al. 2004). Formation of schemas is a central event in student-centered active learning, by which mental models are built of whatever is being learned, consciously and deliberately testing those models to determine whether they work, and then repairing those models that appear to be faulty (Regehr and Norman 1996; Michael 2006). Students learning in this way are more likely to be achieving meaningful learning. Michael has presented evidence supporting active meaningful learning that includes: (a) Learning involves the active construction of meaning by the learner, (b) Learning facts and learning how to do something are two different processes, and, (c) Some things that are learned are specific to the domain or context in which they were learned, whereas other things are more readily transferred to other domains (Michael 2006). This elaboration by active learning opens up the possibility for reflection and better understanding (Patel et al. 2008).

These insights from cognitive psychology on prior knowledge in understanding have relevance for the education process, as they can inform educators about best teaching and learning practices and their impact on the evaluation process (Regehr and Norman 1996; Patel et al. 2008). Teaching of science implies a task of understanding how things work (Mayer 2004). Testing of conceptual understanding will require deeper forms of 
assessment than those used in traditional evaluations. The above mentioned insights also involve student learning, performance and professional competence, as well as recommendations for reform of medical curricula (Patel et al. 2008). Student learning of science involve changing one's mental model or schema how something works, which involves a conceptual change (Posner et al. 1982). A mental model or schema is a cognitive representation of the functional parts of a system and the cause-and-effect relations showing how a change in the state of one part affects a change in the next one (Mayer 1992). According to Mayer three important steps in conceptual change are: (a) Recognizing an anomaly, realizing that one's current mental model is not able to explain the facts observed, (b) Constructing a new model, that can explain the facts, and (c) Using a new model, that makes and tests predictions of the model in new situations (Mayer 2004). Interestingly, each step can be used as a starting point for teaching and learning: (a) Confronting misconceptions, (b) Providing a concrete analogy, and (c) How to test hypotheses, respectively (Mayer 2004). All of the above considerations are based on facts and insights from research in cognitive psychology that is shifting its focus from decontextualized sterile laboratory tasks to contextualized realistic tasks (Mayer 2004). So, teachers should be more aware of what students learn and how they learn (Eva et al. 1998; Dolmans et al. 2005; Michael 2006; Mayer 2010).

These are goals of evidence-based education, a recent intention promoted by, amongst others, the European Commission (Council of the European Union 2007). Evidence-based education tries to bridge the gap between the practice of learning on the one hand, and the development of learning and educational research on the other hand (Council of the European Union 2007; Pirrie 2001; Slavin 2002). In order to implement these goals it has, for example, been advised to: (a) Review how educational professionals, both practitioners and policy-makers, mediate and apply knowledge in their daily work, (b) Promote a positive culture of evaluation that improves the connection between learning objectives and educational practices; and (c) Share new ways of improving accessibility of all types of evidence, so that they inform research, policy and practice (Council of the European Union 2007). In the practice and research of medical education several of these recommendations have already been implemented as a result of fruitful collaborative efforts such as Best Evidence Medical Practice, which have most probably promoted excellence in medical teaching (Ramani 2006). This will be discussed later.

From the scientific platform created by cognitive psychology and educational psychology including evidence-based education we now will engage in an overture for the translation to cognitive neuroscience. Following the ongoing line of reasoning we will focus on the importance and mechanisms of prior knowledge in understanding. We will try to disentangle various aspects of prior knowledge that could be used as individual components subject to behavioral studies. Results from the behavioral studies subsequently could serve as a starting point for cognitive neuroscientific research, which illustrates the multi-step approach in bridging the gaps between medical education and cognitive neuroscience (see Fig. 1). The aspects of prior knowledge to be discussed are selected for their potential relevance in medical education. For medical education, two types of learning in which the importance of prior knowledge is expressed, are multisensory learning (Mayer 2010) and the acquisition of expertise (Schmidt et al. 1990). These types now will be discussed in more detail.

Meaningful learning occurs when a learner selects relevant material from an array of information, organizes it into a coherent representation in a limited capacity working memory, and integrates it with existing knowledge in long-term memory (Regehr and Norman 1996; Mayer 2001). These steps are involved in multimedia learning in which 
Fig. 1 Synergy between Medical Education and Cognitive Neuroscience can be achieved by creating a multidisciplinary educational platform, in which Cognitive Psychology is included

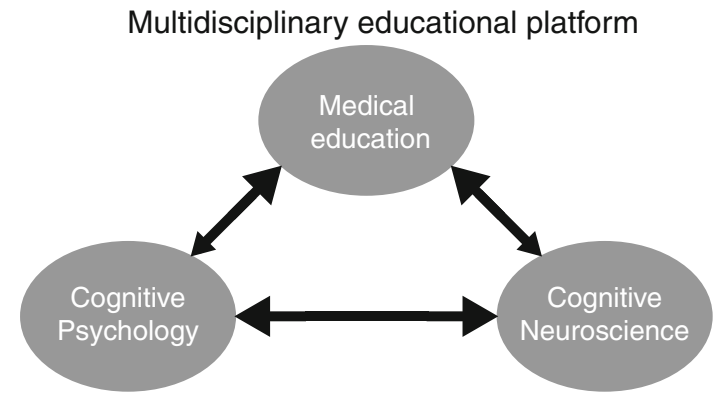

multiple modality information is presented (Mayer 2001). Multiple mental representations can enhance problem-solving abilities, completing each other, resulting in a more authentic portrayal of a problem than any single source of unimodal information (Ainsworth 1999). In line with this, Lasry and Aulls propose a theoretical model of multiple internal mental representations, which they name $n$-coding (Lasry and Aulls 2007).The $n$-coding construct is developed to demonstrate the independence of information processed along different modalities such as verbal, visual, kinesthetic, logico-mathematical, and social modalities. The authors have shown that the construct is useful in designing context-rich environments and can be used to increase learning gains in problem solving, conceptual knowledge, and concept confidence. In addition, it is useful in guiding which additional dimensions in the learning environment need to be attended (Lasry and Aulls 2007).In the rich learning environment in medical education multimodal learning certainly is present. Therefore, a proper insight of the various modalities to meaningful learning in terms of their quality, quantity and sequence (timing) is important. In such a setting, a previous representation would act as prior knowledge for subsequent representations. Multisensory-training protocols, as opposed to unisensory protocols, can better approximate natural settings and therefore, produce greater and more efficient learning (Shams and Seitz 2008). However, the extent to which this facilitation occurs depends on appropriate relations, i.e. congruency, between the information coming into each of the senses. Congruency of new information with prior knowledge can be interpreted as a fit with a schema. More research on multisensory learning paradigms is needed to better understand the mechanisms and processes of learning within natural settings. This also includes the better understanding of the modality effect, the educational practice of presenting to-be-learned graphical information visually, and related textual information through an auditory mode (Ginns 2005). Especially relevant to medical education is multisensory learning through visual and tactile stimuli in order to develop three-dimensional insight of human anatomical structures, and haptic competences for surgical interventions (Miller 2000; Salkini et al. 2010).

Experts are often able to recognize the key features of a problem rapidly, mainly using perceptual cues. This perceptual expertise enables them to be highly selective in their search and to solve routine problems without exploring many alternatives (Gobet 2005).It takes a long time of intense dedication to become an expert- at least ten years in many domains-, including medical specialization and subspecialization (Schmidt et al. 1990). Remarkably, transfer seems to be minimal from one domain of expertise to another. In the development of expert competences schemas are acquired incremently and automatically (Gobet 2005). Gobet and Simon have described a computational model providing a mechanisms explaining how schemas are created in this situation (Gobet and Simon 2000). A schema or template is created if a node meets specific criteria related to its connectivity 
with other nodes in the discrimination network. They are created in situations where the context presents both constant and variable information. An important consequence for education is that without variation of chunks and links connecting them, schemas cannot be created (Gobet 2005). Further implications for education are the difficulty of transferring knowledge from one domain to another, the effect that curriculum sequence may have on learning, the conditions best suited for the acquisition of schemas, and a computational definition for the difficult notion of conceptual understanding. These theoretical considerations from cognitive psychology, as already promoted by Regehr and Norman, foster scientific discussions that may lead to salient issues and questions for research in cognitive neuroscience (Regehr and Norman 1996).

\section{Translation to cognitive neuroscience}

In order to understand processing in the brain and their neural correlates it is important to acquire some of the theoretical background on learning and memory. Miyashita in an excellent review has described the cellular and network machineries of cognitive memory in the brain and their top-down control (Miyashita 2004). The types of explicit memory identified in humans and in animals studied are episodic (event) memory and semantic (fact) memory. The cognitive memory system is composed of three major subsystems, i.e. the medial temporal lobe, the temporal cortex, and the frontal cortex. The medial temporal lobe, which includes the hippocampus, plays a critical role necessary for memory storage to take place. The hippocampus is involved in memory encoding and retrieval (Squire and Alvarez 1995; Wang and Morris 2010). However, the ultimate storage sites for explicit memories appear to be in the neocortex (Miyashita 2004; Wang and Morris 2010). The unique configurational association between environmental stimuli and behavioral context, which is likely the basis of episodic memory, depends on neural circuits in the medial temporal lobe. Memory traces representing repeated associations likely are the basis for semantic memory that is consolidated in domain-specific regions in the temporal cortex. These regions are reactivated during remembering and contribute to the contents of a memory. Two types of retrieval signal reach the cortical representations, one runs from the frontal cortex for active retrieval (top-down), and the other spreads backward from the medial temporal lobe for automatic retrieval. The existence of top-down signaling from the frontal cortex to the temporal cortex was directly demonstrated in monkeys (Miyashita 2004). The challenge is to clarify hierarchical interactions or couplings between multiple cortical areas as initially demonstrated by an effective connectivity analysis or a correlation analysis with functional Magnetic Resonance Imaging (fMRI) in humans. The fMRI technique is now discussed in more detail as it is highly instrumental in cognitive neuroscience and may be so in research on learning.

A wide range of fMRI study designs is available for research in cognitive neuroscience, as elucidated in a comprehensive review by Amaro and Barker (2006). The strategy in an fMRI experiment is based on an intervention in the brain and observation of the modulation of the system response. The MRI method most often used to produce information related to brain function is called BOLD (Blood Oxygenation Level Dependent), and is based on MR images made sensitive to changes in the state of oxygenation of the hemoglobin. Areas with a high concentration of fully saturated oxygen give a higher signal than areas with a low concentration. The practical implication is that BOLD signals are an indirect measure of the increase in neuronal activity at the moment a subject performs a particular task while the person is inside the scanner. BOLD sensitivity is directly 
proportional to the magnetic field strength. Every one to three-seconds images are made and the brain areas in which the brightness changes relative to the task can then be determined using statistical analyses. For neuroimaging other techniques such as positron emission tomography (PET) may be used but the discussion of these techniques is beyond the scope of this paper. Before embarking on neuroimaging it is important to realize whether the scientific question may or may not be suitable for this technique (Amaro and Barker 2006). Therefore, ideally a working hypothesis including a neuroanatomical substrate has to be formulated, and adaptations in the cognitive task experiment have to be made that make it suitable for the neuroimaging environment. Several designs are possible of which the factorial and parametric designs are most commonly used, both single and combined. In the first mentioned design cognitive conditions are processed in a factorial manner, thus allowing tests for interactions between each component. This technique relies upon neuropsychological evidence for precise definition of the task components, and if possible, complementary behavioral data. The principle is to have the participant perform a task when the cognitive components are intermingled in one moment, and separated in another instance of the paradigm (Amaro and Barker 2006). The idea of increasing the cognitive demand associated with a particular cognitive task, without modifying its intrinsic nature is the basis of parametric design. Stimulus presentation strategies include the blocked design, the event-related design, and a mixed design. In the blocked design the stimuli of the same condition are presented subsequently, and the BOLD response represents a steady state actually composed from individual hemodynamic response functions from each stimulus, and is generally of higher magnitude. In the event-related design each stimulus hemodynamic response function(s) is detected, and can be analyzed in detail. Finally, the mixed design consists of a combination of events closely presented, intermingled with control condition providing the technical needs for analysis event-related analysis as well as cognitive state information. The various image acquisition techniques and additional computational exercises to analyze brain connectivity are beyond the scope of this paper.

Now we would like to discuss prior knowledge and learning against the background of the hippocampal-neocortical interactions theory (Morris 2006; Wang and Morris 2010). This theory attempts to map existing neuropsychological ideas about the determinants of episodic memory onto the neural circuits and synaptic processes in both hippocampus and neocortex that have been identified as relevant to memory formation. It encompasses the following important elements: (a) The critical role of mental models or schemas in systems consolidation, i.e. consolidation of memory traces in the neocortex, and (b) Memory updating as a key factor for memory consolidation in the hippocampus. Based on elegant studies in rats (Tse et al. 2007) found that prior knowledge on the correct location in a so called complex event arena plays a causal role in system consolidation. They also showed that system consolidation using a schema based on prior knowledge occurs rapidly, i.e. within $48 \mathrm{~h}$ in this study. This contrasts with the previously widely accepted opinion that it takes a long time before intercortical connections become strong enough to support unaided memory retrieval. The crucial new insight from these results is that new information processed by the hippocampus can be consolidated into the cortex more rapidly if this new information is relevant to prior knowledge, i.e. a schema in which the new information can be assimilated (Tse et al. 2007). This implies that the hippocampus is only required for rapidly updating any new event in relation to these schemas (Wang and Morris 2010). This updating may occur through memory reconsolidation, i.e. the process by which the act of memory retrieval appears to destabilize previously stored memory traces and 
thereby enable them to be strengthened or to incorporate new information (Wang and Morris 2010).

Reports of multisensory interactions in various perceptual tasks and settings indicate that these interactions are the rule rather than the exception in human processing of sensory information (Shimojo and Shams 2001). There exists a rapidly growing literature of the neuroanatomical, electrophysiological and neuroimaging studies that show that multisensory interactions can occur throughout processing (Calvert et al. 2004). According to Shams and Seitz multisensory facilitation of unisensory learning occurs, based on the observation that the learning advantage of auditory-visual training over visual-alone training was substantial (Shams and Seitz 2008). Similar results were found by Von Kriegstein and Giraud, showing that implicit multisensory associations influence voice recognition (Von Kriegstein and Giraud 2006). Regarding the implications and neural mechanisms involved they state that even under conditions of unimodal sensory input, crossmodal neural circuits that have been shaped by previous associative learning become activated and underpin a performance benefit. The key difference between unisensory and multisensory training exists during encoding, whereby a larger set of processing structures are activated in the multisensory paradigms. Shams and Seitz present three possible neural mechanisms as an explanation for multisensory facilitation of unisensory learning. These mechanisms comprise of: (a) Alteration of unisensory structures, (b) Alteration of multisensory structures that interact directly, and (c) Alteration of multisensory structures that are coordinated by a separate multisensory structure. An important aspect of multisensory facilitation of unisensory learning is that facilitation is more pronounced in the congruent condition (Shams and Seitz 2008). The degree of congruency thereby is clearly relevant for learning. This means that the relationship between the stimuli that are consistent with the prior experience of the individual or the relationships between the senses found in nature is important to facilitate learning (Shams and Seitz 2008). Recent results from our group (Van Kesteren et al. (2010b) manuscript under revision) demonstrate that successful retrieval of congruent compared to incongruent visuo-tactile associations is related to enhanced processing in a medial prefrontal somatosensory network. This suggests optimized memory consolidation by facilitation of neocortical integration when new information fits a preexisting schema. Further research is needed to study how the integration of complex multisensory object information in the human neocortical networks takes place.

Recent studies suggest that the semantic relationship between multimodal input streams might be a relevant factor in the way different areas are recruited during multimodal integration (Willems et al. 2009). Willems et al. report that the integration of speech and co-speech gestures in the (pre) motor cortical brain areas involved represent the matching of two input streams for which there is a relatively stable common object representation, whereas integration in the prefrontal cortex is better characterized as the on-line construction of a new and unified representation of the input streams (Willems et al. 2009). Co-speech gestures embody a form of manual action that is tightly coupled to the language system. Interestingly, the (pre) motor cortex can be activated to action words (Willems and Hagoort 2007). Ruschemeyer et al. (2007) compared activations to action verbs with "complex verbs" that had the action verb as its stem, but did not have an action-related meaning. For example, the German verb "greifen" (grasp) was compared to "begreifen" (to understand, to comprehend). The rationale was that if action verbs automatically activate parts of the motor cortex, this should also be the case in complex verbs that include an action verb, such as "begreifen". They found that action words did activate premotor cortical areas compared to abstract verbs. No such activation was however found in response to the complex verbs. It is not well known whether complex verbs such as 
"begreifen" are stored with "greifen" (to grasp) as their stem. Still, these intriguing findings raise an interest to further study the neural mechanisms of embodiment, as they may have relevance for multimodal learning. In a much broader sense, the heart of the matter of understanding could be to embody that matter (McVee et al. 2004; Wilson 2002).

\section{Opportunities for medical education and medical education research}

The overall goals of medical education are (a) The acquisition of the knowledge, skills, attitudes and values required to perform professional medical tasks competently and safely, and (b) The development and continuous refinement of the basic clinical skills that are required to provide competent care throughout a lifetime of professional work (AAMC Report 2004; AAMC Report 2005). As indicated by Regehr and Norman (1996), and Patel et al. (2008) and already touched on in the previous paragraphs cognitive and learning sciences theories have great relevance in informing the process of medical education evaluation, including its impact on student learning, performance and professional competence, as well as recommendations for reform of medical curricula based on such theories. This includes the insights from cognitive psychology on prior knowledge in understanding on the one hand, and the attempts to translate these to cognitive neuroscience on the other hand. Against this background we now will illustrate how such integrative approach may create opportunities for medical education. In order to create such opportunities it is important for medical education to formulate firm ambitions in terms of excellence and to set high standards for medical education research (Ramani 2006; Schuwirth and Van der Vleuten 2006). Aspects of medical education and those of medical education research will be discussed separately.

Cognitive neuroscience has driven educational progress, that also is highly relevant for medical education (Goswami 2006). The discovery of mirror neurons has enforced the insight that imitating helps learning (Gazzola et al. 2007). Excellent teachers that act as role models may enhance learning this way (Ramani 2006). The recognition of prolonged brain development during adolescence has consequences for learning and teaching of our, especially male bachelor students, as their integrative cerebral functions have not fully matured yet (Draganski et al. 2006). On the other hand, brain plasticity may induced by extensive learning of medical students (Bosma et al. 2002), and it may happen until older age (Karp et al. 2006), yielding good physiological perspectives for long life learning (Bosma et al. 2002; Karp et al. 2006; Wilson et al. 2002), which is an important feature of keeping up professional medical competences. Experiences are better stored when learned in an emotional or arousing setting and increased motivation (Squire and Alvarez 1995), which is often the case in medical education. The presence of multiple intelligences necessitates splitting up intelligences helping developing one at a time (Gardner and Hatch 1989). Insights into consciousness in the brain helps to analyze learning (Gardiner 2001). Last but not least, these new findings and insights driven by cognitive neuroscience have enriched all the biomedical disciplines that deal with normal brain function and structure and their clinical counterparts. This implies integration of recent advances in neuroscience into e.g. undergraduate neuroscience and physiology courses (Cleland 2002). In addition, research training on psychiatry and neurosciences to medical students has been introduced (Balon et al. 2006).

In order to reach excellence in medical education Ramani proposed a number of recommendations, including establishing explicit learning and teaching outcomes, implementing best evidence medical education (BEME), and evaluating teaching and impact of 
teaching (Ramani 2006). These recommendations not only have great relevance for the insight in learning efficacy of students, but they also challenge the teaching faculty in its role of an educational scholar that is interested in what students learn and how they learn. This scholarly attitude is a prerequisite to be able to create opportunities to use insights from cognitive psychology in understanding student's learning and to be able to improve the curriculum on a scientifically sound basis. Assessment of learning efficacy provides a means to obtain consistent data to feed curriculum improvement and to formulate questions for educational research. Assessment in medical education currently involves an integrated approach of formative and summative assessments and regular evaluation of competences that is recorded in a student portfolio (Schuwirth and Van der Vleuten 2006; Epstein 2007). An important goal of assessment is to optimize the capabilities of all learners and practicioners by providing motivation and direction for future learning (Epstein 2007). Such assessment thus can be viewed as an educational tool that provides useful information for both students and faculty (Krupat and Dienstag 2009). Recently, the role of interim assessments a third type of assessment in a comprehensive assessment system of US school districts was described, that (a) Evaluate student's knowledge and skills relative to a specific set of academic goals, typically within a limited time frame, and (b) Are designed to inform decisions at both the classroom and beyond the classroom level (Perie et al. 2007). As the results of interim assessments can be meaningfully aggregated and reported at a broader level they could serve as input for evidence-based education that could be useful for scientific elaboration. In addition, repeated assessments may enhance learning, known as the testing effect (Larsen et al. 2008). So, next to the scholarly attitude of the teaching faculty, data generated by interim assessments could involve the teachers in scientific discussions with peers, students, and cognitive psychologists. Translation of salient issues and questions to cognitive neuroscience can be stimulated by structured discussions that take place in a multidisciplinary educational research platform (Fig. 1). This is an extension of the close collaboration between doctors and educationalists as proposed by Schuwirth and Van der Vleuten (Schuwirth and Van der Vleuten 2006). It is important to share new ways of improving accessibility of all types of evidence, so that they inform research, policy, and practice (Council of the European Union 2007).

Research on medical education, especially problem-based learning (Norman and Schmidt 1992), should contribute to a better understanding of why and how the concepts of constructive, self-directed, collaborative and contextual learning do or do not work and under which circumstances (Dolmans et al. 2005). As we have elucidated in previous paragraphs, prior knowledge built in a schema, greatly facilitates contextual learning if the new information can be assimilated in the schema. Medical education makes use of several schemas in which knowledge and understanding is constructed and reconstructed and they are of different magnitude (Regehr and Norman 1996). Such schemas may vary from the three dimensional structure of an organ, to the complex function of (a component of) an organ, to a certain reaction pattern characteristic to a group of diseases, and, to the rationale of a diagnostic or therapeutic intervention. It would be fascinating to understand the critical conditions to build a biomedical schema, and to reconstruct it following assimilation of new knowledge. This may be highly relevant for the integration between clinical experiences and the basic science courses (Norman 2007; Patel et al. 2008), and for the process of illness script formation (Norman 2005; Schmidt and Rikers 2007). Related questions include: Does the degree of congruency with prior knowledge influence learning efficacy? Does the type of schema, e.g. unimodal or multimodal, influence the neural mechanism of processing and coordination? How does multimodal learning enhance insight into three dimensional proportions such as in human anatomy (Marks 2000)? Which neocortical 
areas are involved in schema consolidation and in which hierarchy (Miyashita 2004; Wang and Morris 2010)? Which factors interfere with schema formation? It would be very challenging seeing the learning efficacy in educational conditions in which schema driven learning is manipulated in order to improve learning efficacy. Related questions include: Is there a difference in learning effect of a meaningful schema and a memory aid schema? What is the optimal time interval to add new knowledge to a schema? What is the maximal qualitative (complexity) and quantity (load/overload) of a schema? How long does a schema lasts and in which condition (decay?)? How are schemas reconstructed during the development of expertise? Can embodiment enhance schema formation? Does schema formation concern both explicit and implicit memory? How can we avoid incoherence of new knowledge with a schema, leading to interference with learning as observed in multitasking and repetitive task shifting (Small and Vorgan 2008)? Can we use schemas as connecting elements in designing or remodeling individual courses, series of courses, or curricula? Can they be related to concrete learning objectives as used in a learner outcome oriented curriculum? And, can the use of schemas facilitate problem formulation by helping students to recognize the nature of the problem (Auclair 2007)?

We hope that the fruits of these research efforts may strengthen the bridges between medical education and cognitive neuroscience, thereby achieving synergy. And like Einstein we believe that integrated research of the two domains should be driven by an ongoing urge to understand (Einstein 1934).

\section{Conclusions and recommendations}

From the foregoing discussion the following conclusions and recommendations can be made:

1. Based on the promising results of initial integrative research between cognitive neuroscience and education research, and the great potential for medical education and medical education research, synergy between medical education and cognitive neuroscience should be possible.

2. Cognitive psychology plays an important role as a scientific interface between medical education and cognitive neuroscience.

3. As formation of schemas is a central event in student-centered active learning and has relevance for both medical education and cognitive neuroscience the topic seems particularly suitable for integrative research efforts.

4. (Medical) teachers should be more aware of what students learn and how they learn, which are goals of evidence-based education that tries to bridge the gap between the practice of learning on the one hand, and the development of learning and educational research on the other hand.

5. In order to understand processing in the brain and their neural correlates it is important to acquire some of the theoretical background on learning and memory. Before embarking on neuroimaging, it is important to realize whether the scientific question may or may not be suitable for the different imaging techniques.

6. Recent neurocognitive research in human subjects shows evidence for multisensory facilitation of unisensory learning, which facilitation is specific to the congruent condition. Congruency between stimuli may reflect whether they can be readily integrated in a schema. 
7. The dialogue on interesting research alleys on the crossroad of medical education including medical education research, and cognitive neuroscience should proceed along a research agenda designed by a multidisciplinary educational research platform, in which cognitive psychology is included.

8. In order to increase opportunities for this research the medical educational field should further promote evidence-based education in order to create a platform for having an optimal dialogue.

9. Instead of bridging preexisting research domains a new learning-centered domain could be opened: a field of mind, brain, and education, or, alternatively, of educational psychology, or even a new science of learning.

10. Opportunities to study interesting research topics in medical education should be explored, e.g. the role of prior knowledge in understanding, multimodal learning, and the development of expertise.

11. Integrative training to professionals with interdisciplinary competences in the domain of mind, brain and education, who share the same language and theoretical frameworks, is strongly advocated.

Acknowledgments The authors would like to thank Dr. Jan Kooloos, Department of Anatomy, and Professor Jan van der Eerden and Dr. Mark Rijpkema, Donders Center for Cognitive Neuroimaging, for giving valuable comments.

Open Access This article is distributed under the terms of the Creative Commons Attribution Noncommercial License which permits any noncommercial use, distribution, and reproduction in any medium, provided the original author(s) and source are credited.

\section{References}

AAMC. (2004). Educating doctors to provide high quality medical care: A vision for medical education in the United States. Report of the Ad Hoc Committee of Deans. Washington, DC. Accessible through https://services.aamc.org/publications/showfile.cfm?file=version27.pdf\&prd_id=1158prv_id=1308pdf_ $\mathrm{id}=27$.

AAMC. (2005). Recommendations for clinical skills curricula for undergraduate medical education. Accessible through https://services.aamc.org/Publications/index.cfm?fuseaction=Product.displayForm\& prd_id=141\&prv_id=165.

Ainsworth, S. (1999). The functions of multiple representations. Computational Education, 33, 131-152.

Alba, W. J., \& Hasher, L. (1983). Is memory schematic? Psychological Bulletin, 93, 203-231.

Amaro, E. Jr., \& Barker, G. J. (2006). Study design in fMRI: Basic principles. Brain Cognition, 60, 220-232.

Ansari, D., \& Coch, D. (2006). Bridges over troubled waters: Education and cognitive neuroscience. Trends in Cognitive Science, 10, 146-151.

Auclair, F. (2007). Problem formulation by medical students: An observation study. BMC Medical Education, 7, 16. doi:10.1186/1472-6920-7-16.

Balon, R., Heninger, G., \& Belitsky, R. (2006). Medical school research pipeline: Medical student research experience in psychiatry. Academic Psychiatry, 30, 16-22.

Bosma, H., Van Boxtel, M. P. J., Ponds, R. W. H. M., Jelicic, M., Houx, P. J., Metsemakers, J., et al. (2002). Engaged lifestyle and cognitive function in middle and old-aged, non-demented persons: A reciprocal association? Zeitschrift fuer Gerontologie und Geriatrie, 35, 575-581.

Bransford, J. D. (1983). Schema activation and schema acquisition: Comments on Richard C Anderson's remarks. In R. C. Anderson, J. Osborn, \& R. C. Tierney (Eds.), Learning to read in American schools (pp. 259-272). Hillsdale, NJ: Lawrence Erlbaum.

Bransford, J. D., \& Johnson, M. K. (1972). Contextual prerequisites for understanding: Some investigations of comprehension and recall. Journal of Verbal Learning and Verbal Behavior, 11, 717-726.

Bruer, J. T. (1997). Education and the brain: A bridge too far. Educational Research, 26, 4-16. 
Calvert, G. A., Spence, C., \& Stein, B. E. (Eds.). (2004). The handbook of multisensory processes. Boston: MIT Press.

Christodoulou, J. A., \& Gaab, N. (2009). Using and misusing neuroscience in education-related research. Cortex, 45, 555-557.

Cleland, C. L. (2002). Integrating recent advances in neuroscience into undergraduate neuroscience and physiology courses. Advances in Physiology Education, 26, 271-277.

Coch, D., \& Ansari, D. (2009). Thinking about mechanisms is crucial to connecting neuroscience and education. Cortex, 45, 546-547.

Council of the European Union. (2007). Commission staff working document: Towards more knowledgebased policy and practice in education and training. Brussels. Accessible through http://www.eu/ policies/2010/doc/sec/1098.

De Jong, T., Van Gog, T., Jenks, K., Manlove, S., Van Hell, J. G., Jolles, J., et al. (2008). Explorations in learning and the brain: On the potential of cognitive neuroscience for educational science. Report by the Netherlands Organisation for Scientific Research. The Hague, The Netherlands. Accessible through http://www.eric-project.nl/nwohome.nsf/pages/NWOA_7GJDST.

Della Salla, S. (2009). Editorial. The use and misuse of neuroscience in education. Cortex, 45, 443.

Dolmans, D. H., De Grave, W., Wolfhagen, I. H., \& Van der Vleuten, C. P. (2005). Problem-based learning: Future challenges for educational practice and research. Medical Education, 39, 732-741.

Draganski, B., Gaser, C., Kempermann, G., Kuhn, H. G., Winkler, J., Buechel, C., et al. (2006). Temporal and spatial dynamics of brain structure changes during extensive learning. Journal of Neuroscience, 26, 6314-6317.

Einstein, A. (1934). The world as I see it. Originally published in 1934. Yucca Valley: Citadel Publ 2001.

Epstein, R. M. (2007). Assessment in medical education. New England Journal of Medicine, 356, 387-396.

Eva, K. W. (2005). What every teacher needs to know about clinical reasoning. Medical Education, 39, 98-106.

Eva, K. W., Neville, A. J., \& Norman, G. R. (1998). Exploring the etiology of content specificity: Factors influencing analogic transfer and problem solving. Academic Medicine, 73, S1-S5.

Gardiner, J. M. (2001). Episodic memory and autonoetic consciousness, a first-person approach. Philosophical Transactions of the Royal Society, London, Series B-Biological Sciences, 356, 1351-1361.

Gardner, H., \& Hatch, T. (1989). Educational implications of the theory of multiple intelligences. Educational Research, 18, 4-10.

Gazzola, V., Rizzolatti, G., Wicker, B., \& Keysers, C. (2007). The anthropomorphic brain: The mirror neuron system responds to human and robotic actions. Neuroimage, 35, 1674-1684.

Ginns, P. (2005). Meta-analysis of the modality effect. Learning and Instruction, 15, 313-331.

Gobet, F. (2005). Chunking models of expertise: Implications for education. Applied Cognitive Psychology, 19, 183-204.

Gobet, F., \& Simon, H. A. (2000). Five-seconds or sixty? Presentation time in expert memory. Cognitive Science, 24, 651-682.

Goswami, U. (2006). Neuroscience and education: From research to practice? Nature Reviews Neuroscience, 7, 406-411.

Greenwood, R. (2009). Where are the educators? What is our role in the debate? Cortex, 45, 552-554.

Karp, A., Paillard-Borg, S., Wang, H., Silverstein, M., Winblad, B., \& Fratiglioni, L. (2006). Mental, physical and social components in leisure activities equally contribute to decrease dementia risk. Dementia Geriatric Cognitive Diseases, 21, 65-73.

Krupat, E., \& Dienstag, J. L. (2009). Assessment is an educational tool. Academic Medicine, 84, 548-550.

Larsen, D. P., Butler, A. C., \& Roediger, H. L. III. (2008). Test-enhanced learning in medical education. Medical Education, 42, 959-966.

Lasry, N., \& Aulls, M. W. (2007). The effect of multiple internal representations on context-rich instruction. American Journal of Physics, 75, 1030-1037.

Marks, S. C. Jr. (2000). The role of three-dimensional information in health care and medical education: The implications for anatomy and dissection. Clinical Anatomy, 13, 448-452.

Mason, L. (2009). Bridging neuroscience and education: A two-way path is possible. Cortex, 45, 548-549.

Mayer, R. E. (1992). Thinking, problem solving, cognition (2nd ed.). New York: Freeman.

Mayer, R. E. (2001). Multimedia learning. Cambridge: Cambridge University Press.

Mayer, R. E. (2004). Teaching of subject matter. Annual Review of Psychology, 55, 715-744.

Mayer, R. E. (2010). Applying the science of learning to medical education. The cross-cutting edge. Medical Education, 44, 543-549.

McVee, M. B., Dunsmore, K., \& Gavelek, J. R. (2004). Schema theory revisited. Review of Educational Research, 75, 531-566. 
Meltzoff, A. N., Kuhl, P. K., Movellan, J., \& Sejnowski, T. J. (2009). Foundations for a new science of learning. Science, 325, 284-288.

Michael, J. (2006). Where's the evidence that active learning works? Advances in Physiology Education, 30, 159-167.

Miller, R. (2000). Approaches to learning spatial relationships in gross anatomy: Perspectives from wider principles of learning. Clinical Anatomy, 13, 439-443.

Miyashita, Y. (2004). Cognitive memory: Cellular and network machineries and their top-down control. Science, 306, 435-440.

Morris, R. G. M. (2006). Elements of a neurobiological theory of hippocampal function: The role of synaptic plasticity, synaptic tagging and schemas. European Journal of Neuroscience, 23, 2829-2846.

Neville, A. J., \& Norman, G. R. (2007). PBL in the undergrate MD program at McMaster University: Three iterations in three decades. Academic Medicine, 82, 370-374.

Norman, G. R. (2005). Research in clinical reasoning: Past history and current trends. Medical Education, $39,418-427$.

Norman, G. R. (2007). How basic is basic science? Advances in Health Sciences Education, 12, 401-403.

Norman, G. R., \& Schmidt, H. G. (1992). The psychological basis of Problem-based Learning: A review of the evidence. Academic Medicine, 67, 557-565.

Patel, V. L., Yoskowitz, N. A., \& Arocha, J. F. (2008). Towards effective evaluation and reform in medical education: A cognitive and learning science perspective. Advances in Health Science Education. doi: 10.1007/s10459-007-9091-1.

Perie, M., Marion, S., Gong, B., \& Wurtzel, J. (2007).The role of interim assessments in a comprehensive assessment system. Measures that matter. The Aspen Institute. Accessible through http//www.achieve. org/files/TheRoleofInterimAssessments.pdf.

Perkins, D. (2009). On grandmother neurons and grandfather clocks. Mind, Brain and Education, 3, $170-175$.

Pirrie, A. (2001). Evidence-based practice in education: The best medicine? British Journal for Educational Studies, 49, 124-136.

Posner, G. J., Strike, K. A., Hewson, P. W., \& Gertzog, W. A. (1982). Accomodation of scientific conception: Toward a theory of conceptual change. Scientific Education, 66, 211-227.

Quellmalz, E. S., \& Pellegrino, J. W. (2009). Technology and testing. Accessible through www.sciencemag. org/education_technology 2009.

Ramani, S. (2006). Twelve tips to promote excellence in medical teaching. Medical Teacher, 8, 19-23.

Regehr, G., \& Norman, G. R. (1996). Issues in cognitive psychology: Implications for professional education. Academic Medicine, 71, 988-1001.

Ruschemeyer, S.-A., Brass, M., \& Friederici, A. D. (2007). Comprehending prehending: Neural correlates of processing verbs with motor stem. Journal of Cognitive Neuroscience, 19, 855-865.

Salkini, M. W., Doarn, C. R., Kiehl, N., Broderick, T. J., Donovan, J. F., \& Gaitond, K. (2010). The role of haptic feedback in laparoscopic training using the LapMentor II. Journal of Endourology, 1, 99-102.

Schmidt, H. G., Norman, G. R., \& Boshuizen, H. P. A. (1990). A cognitive perspective on medical expertise: Theory and implications. Academic Medicine, 65, 611-621.

Schmidt, H. G., \& Rikers, R. M. J. P. (2007). How expertise develops in medicine: Knowledge encapsulation and illness script formation. Medical Education, 41, 1133-1139.

Schuwirth, L. W. T., \& Van der Vleuten, C. P. M. (2006). Medical education. Challenges for educationalists. British Medical Journal, 333, 544-546.

Shams, L., \& Seitz, A. R. (2008). Benefits of multisensory learning. Trends in Cognitive Sciences, 12, 411-417.

Shimojo, S., \& Shams, L. (2001). Sensory modalities are not separate modalities: Plasticity and interactions. Current Opinion in Neurobiology, 11, 505-509.

Slavin, R. E. (2002). Evidence-based education policies: Transforming educational practice and research. Educational Research, 31, 15-21.

Small, G., \& Vorgan, G. (2008). iBrain: Surviving the technological alteration of the modern mind. New York: Collins Living.

Squire, L. R., \& Alvarez, P. (1995). Retrograde amnesia and memory consolidation: A neurobiological perspective. Current Opinion in Neurobiology, 5, 169-177.

Takashima, A., Nieuwenhuis, I. L., Jensen, O., Talamini, L. M., Rijpkema, M., \& Fernandez, G. (2009). Shift from hippocampal to neocortical centered retrieval network with consolidation. $J$ Neuroscience, 29, 10087-10093.

Takashima, A., Petersson, K. M., Rutters, F., Tendolkar, I., Jensen, O., Zwarts, M. J., et al. (2006). Declarative memory consolidation in humans: A prospective functional magnetic resonance imaging study. Proceedings of the National Academy of Sciences USA, 103, 756-761. 
TLRP Commentary. (2008). Neuroscience and education: Issues and opportunities. Accessible through www.tlrp.org.

Tse, D., Langston, R. F., Kakeyama, M., Bethus, I., Spooner, P. A., Wood, E. R., et al. (2007). Schemas and memory consolidation. Science, 316, 76-82.

Van Kesteren, M. T., Fernandez, G., Norris, D. G., \& Hermans, E. J. (2010a). Persistent schema-dependent hippocampal-neocortical connectivity during memory encoding and postencoding rest in humans. Proceedings of the National Academy of Sciences USA, 107, 7550-7555.

Van Kesteren, M. T., Rijpkema, M., Ruiter, D. J., \& Fernandez, G. (2010b). Retrieval of associative information congruent with prior knowledge is related to increased medial prefrontal activity and connectivity. Manuscript under revision.

Varma, S., McCandliss, B. D., \& Schwartz, D. L. (2008). Scientific and pragmatic challenges for bridging education and neuroscience. Educational Research, 37, 140-152.

Von Kriegstein, K., \& Giraud, A.-L. (2006). Implicit multisensory associations influence voice recognition. PLoS Biology, 4, 1806-1820.

Wang, S.-Z., \& Morris, R. G. M. (2010). Hippocampal-neocortical interactions in memory formation, consolidation, and reconsolidation. Annual Review of Psychology, 61, 49-79.

Willems, R. M., \& Hagoort, P. (2007). Neural evidence for the interplay between language, gesture, and action: A review. Brain and Language, 101, 278-289.

Willems, R. M., Ozyurek, A., \& Hagoort, P. (2009). Differential roles for left inferior frontal and superior temporal cortex in multimodal integration of action and language. Neuroimage, 47, 1992-2004.

Wilson, M. (2002). Six views of embodied cognition. Psychoneurological Bulletin Review, 9, 625-636.

Wilson, R. S., Mendes de Leon, C. F., Barnes, L. L., Schneider, J. A., Bienias, J. L., Evans, D. A., et al. (2002). Participation in cognitively stimulating activities and risk of incident Alzheimer disease. Journal of the American Medical Association, 287, 742-748. 